\title{
Direct Multiple Shooting Optimization with Variable Problem Parameters
}

\author{
Ryan J. Whitley ${ }^{*}$ \\ NASA Johnson Space Center, Houston, Texas 77062 \\ Cesar A. Ocampo ${ }^{\dagger}$ \\ University of Texas, Austin, Texas 78712
}

\begin{abstract}
Taking advantage of a novel approach to the design of the orbital transfer optimization problem and advanced non-linear programming algorithms, several optimal transfer trajectories are found for problems with and without known analytic solutions. This method treats the fixed known gravitational constants as optimization variables in order to reduce the need for an advanced initial guess. Complex periodic orbits are targeted with very simple guesses and the ability to find optimal transfers in spite of these bad guesses is successfully demonstrated. Impulsive transfers are considered for orbits in both the 2-body frame as well as the circular restricted three-body problem (CRTBP). The results with this new approach demonstrate the potential for increasing robustness for all types of orbit transfer problems.
\end{abstract}

\section{Introduction}

Solving complex trajectory problems optimally is of vital importance to the success of future aerospace programs. For example, NASA is currently engaged in developing a new human spaceflight program to the Moon that places new complex constraints on the spacecraft trajectories there and back. This is only one example; other interplanetary missions are being developed and will lead to new programs that will continue to expand out into the universe. If we are to succeed in these endeavors, our ability to find optimal trajectories must expand with it.

In the ever growing field of optimization research, algorithm robustness is the ultimate aim. One approach to achieve this robustness is to modify current optimization approaches to handle complex problems without requiring meticulous tuning to obtain a solution; in other words, construct an algorithm that does not require a feasible target solution as a starting point or advanced knowledge of the direction of the optimal solution.

Through the ages, trajectory optimization research has been divided into two main categories. Both methodologies have been tested and extensively applied to specific orbital trajectory

* GNC Engineer, Aeroscience and Flight Mechanics Division, NASA JSC, 2101 Nasa Pkwy. Houston, Texas 77058 / EG-5, AIAA Member

${ }^{\dagger}$ Associate Professor, Department of Aerospace Engineering and Engineering Mechanics, The University of Texas at Austin, 210 E. $24^{\text {th }}$ St., Austin, TX 78712, AIAA Member. problems. Indirect methods take advantage of calculus of variations techniques to solve two-point boundary problems. Direct methods transform the continuous problem into a parameter optimization problem which is solved numerically. Many approaches to the direct problem have been developed including implicit approximation of the states as well as explicit integration techniques, known as the direct shooting approach.

The algorithm presented here employs the second type of direct trajectory optimization, but with a new twist. The equations of motions that dictate the behavior of bodies in space have fixed constants. These constants may be invariable, but that does not mean that they cannot be treated as optimization variables. The constants can be constrained to the fixed values, thus imparting a target error on known values and ensuring the final solution is feasible.

\section{$\boldsymbol{\mu}$ as an Optimization Parameter}

For orbit dynamicists, the primary constant that appears in almost all calculations is the gravitational parameter, determined individually for each massive body in the solar system. Imagine treating this parameter as a variable. In the simplest example, a two-body system, the force model is given by the following equation of motion:

$$
\ddot{\vec{r}}=-\frac{\mu \vec{r}}{r^{3}}
$$

The dynamics in this system is controlled entirely by the value of this parameter. If the parameter is very large, like the sun, the force exerted on the spacecraft is equally large. But if the gravitational constant were very small this force is 
similarly smaller and less influential. In fact, if the parameter was small enough to be infinitesimally tiny, a force-free field is created. In this system, an optimal trajectory has an obvious structure: a straight line.

It is this fact that the algorithm attempts to exploit. Start with an easily constructed straight line guess in a field free system and the algorithm adapts the solution to the unique quality of the problem by changing values of the gravitational parameter in the direction of the known constant. If the solution process from iteration to iteration is modified gradually to the desired value, the optimization routine may be able to handle very complex problems.

In applying this technique to more complex systems, such as the circular restricted three-body problem (CRTBP), the gravitational parameter has a different meaning and a straight line guess is no longer precisely a solution at any phase of the problem. For example, in the CRTBP $\mu$ is now a ratio of gravitational parameters, the secondary over the primary body. A value of zero simply eliminates the secondary body from the problem, the primary body is still present and thus a force-free field space can never exist. However, this fact does not necessarily discount the usefulness of treating the parameter as an optimization variable in the CRTBP frame.

The CRTBP system is useful tool for mission design because of the presence of periodic orbits that subsist solely due to the interaction of the two bodies. If the gravitational parameter changes these periodic orbits breakdown. However, transfers to these orbits typically take advantage of the unstable manifolds associated with these orbits, so the breakdown that occurs with the changing parameter may be useful in zeroing in on an optimal solution.

While a straight line guess is no longer valid it is still a useful starting point. In this instance, this is particularly made true if the number of independent segments is large. The segments can then be splayed out equally between the two targets and along a straight line. Propagating each segment does not deviate very significantly from this line initially.

\section{Direct Multiple Shooting Approach}

The segments in the previous section refer to the multiple shooting arcs in the formulation of the optimization problem. In this particular multiple shooting formulation every segment contains a set of 8 independent variables including initial and final time and 3 components each of position and velocity at the initial time. Since the number of ideal segments might vary from problem to problem the algorithm was designed to handle any $n$ number of them. Each segment thus has the following parameter structure:

$$
\vec{x}_{p_{i}}=\left[\begin{array}{llllllll}
x_{i 0} & y_{i 0} & z_{i 0} & v x_{i 0} & v y_{i 0} & v z_{i 0} & t_{i 0} & t_{i f}
\end{array}\right]^{T}
$$

From this parameter vector, the state is propagated to the final time. Continuity is then ensured not internally but by a constraint parameter vector. The state and time at the endpoints of the segments are ensured to be continuous by equality constraints and time from segment to segment is guaranteed to increase monotonically with inequality constraints:

$$
\vec{c}_{i}=\left[\begin{array}{c}
x_{i 0}-x_{i-1 f} \\
y_{i 0}-y_{i-1 f} \\
z_{i 0}-z_{i-1 f} \\
v x_{i 0}-v x_{i-1 f} \\
v y_{i 0}-v y_{i-1 f} \\
v z_{i 0}-v z_{i-1 f} \\
t_{i 0}-t_{i-1 f} \\
t_{i f}-t_{i 0}
\end{array}\right] \geq\left[\begin{array}{l}
0 \\
0 \\
0 \\
0 \\
0 \\
0 \\
0
\end{array}\right]
$$

Next, constant $\mu$ needs to be included as a parameter. This variable is the same value for each segment, there is not a separate gravity constant for each segment:

$$
\begin{gathered}
\vec{x}_{\mu}=[\mu] \\
\vec{c}_{\mu}=\left[\mu_{\text {tar }}-\mu\right]=0
\end{gathered}
$$

Equations 2 and 3 together form the basic structure for the variables that are used in every formulation of the problem.

The differences occur in the end-point target constraints. However, throughout this paper, the target constraints are the same. They are all position constraints (velocity differences at the endpoints are incorporated in the objective function). Assuming additional time like parameters $\left(\tau_{0}\right.$ and $\left.\tau_{f}\right)$ for the initial and final orbits, the constraints are given below:

$$
\vec{c}_{\text {endpt }}=\left[\begin{array}{c}
x_{i n i}\left(\tau_{0}\right)-x_{10} \\
y_{\text {ini }}\left(\tau_{0}\right)-y_{10} \\
z_{\text {ini }}\left(\tau_{0}\right)-z_{10} \\
x_{n f}-x_{t a r}\left(\tau_{f}\right) \\
y_{n f}-y_{\text {tar }}\left(\tau_{f}\right) \\
z_{n f}-z_{\text {tar }}\left(\tau_{f}\right)
\end{array}\right]=\left[\begin{array}{l}
0 \\
0 \\
0 \\
0 \\
0 \\
0
\end{array}\right]
$$




\section{Formal Problem Definition}

With these constraints and parameters established, it is possible to delineate the entire problem formulation. Since impulsive transfers are the focus of this algorithm, the initial and final $\Delta v$ 's constitute the two parts of the objective function:

$$
J=\Delta v_{0}+\Delta v_{f}
$$

In terms of the parameter vector variables, this is given as follows:

$J=\left[\left(v x_{i n i}-v x_{10}\right)^{2}+\left(v y_{i n i}-v y_{10}\right)^{2}+\left(v z_{i n i}-v z_{10}\right)^{2}\right]^{1 / 2}+$

$\left[\left(v x_{n f}-v x_{\text {tar }}\right)^{2}+\left(v y_{n f}-v y_{\text {tar }}\right)^{2}+\left(v z_{n f}-v z_{\text {tar }}\right)^{2}\right]^{1 / 2}$

And finally the entire parameter vector and constraint vector combined:

$$
\vec{x}_{p}=\left[\begin{array}{c}
x_{10} \\
\vdots \\
v z_{10} \\
t_{10} \\
t_{1 f} \\
\vdots \\
x_{n 0} \\
\vdots \\
v z_{n 0} \\
t_{n 0} \\
t_{n f} \\
\mu \\
\tau_{0} \\
\tau_{f}
\end{array}\right] \quad \vec{c}=\left[\begin{array}{c}
x_{20}-x_{1 f} \\
\vdots \\
v z_{20}-v z_{1 f} \\
t_{20}-t_{1 f} \\
t_{1 f}-t_{10} \\
\vdots \\
x_{n 0}-x_{n-1 f} \\
\vdots \\
v z_{n 0}-v z_{n-1 f} \\
t_{n 0}-t_{n-1 f} \\
t_{n-1 f}-t_{n-10} \\
\mu_{\text {tar }}-\mu \\
x_{i n i}-x_{10} \\
y_{i n i}-y_{10} \\
z_{i n i}-z_{10} \\
x_{n f}-x_{\text {tar }} \\
y_{n f}-y_{\text {tar }} \\
z_{n f}-z_{\text {tar }} \\
t_{10}-0
\end{array}\right]=\left[\begin{array}{l}
0 \\
0 \\
0 \\
0
\end{array}\right]=\left[\begin{array}{l}
0 \\
0 \\
0 \\
0 \\
0 \\
0 \\
0 \\
0 \\
0 \\
0 \\
0 \\
0 \\
0 \\
0
\end{array}\right]
$$

The length of the parameter vector is $8 n+3$. The constraint vector has length $8 n+1.8 n$ of these constraints are inequality while the rest are equality constraints. Since the number of total constraints does not exceed the length of the parameter vector this is a well-defined optimization problem. (The number of variables only has to exceed the number of equality and active inequality constraints, but noting the former difference guarantees this.) It is also worthwhile to note that the inequality constraints will only be active if the segment is reduced to be infinitesimally small $\left(t_{i 0}=t_{i f}\right)$. All of the derivations in this paper are based solely on the parameter and constraint vectors above, but some of the results in the 2 body system use true anomalies in place of $\tau$ 's for the final two parameters; the derivations of these gradients have not been provided here for brevity.

\section{Analytic Gradient Derivation}

In many instances, for the sake of expediency, it is logical to avoid the tedious derivation process necessary to obtain precise analytic derivatives and use a numerical finite difference method instead. This is not one of those instances. While numerical methods can provide very close approximations of the actual derivatives nothing can approach the accuracy of analytical gradients. And while for some simple problems or problems that have good initial guesses these derivatives are adequate, the goal of this formulation is to be robust, and thus analytic gradients are necessary to ensure a truly powerful algorithm.

In addition, with the size of this problem potentially being very large depending on the number of segments selected, the importance of analytic gradients in terms of the efficiency and/or capability in solving a given problem becomes even more critical. With finite differences, each additional variable and constraint adds at least a couple of function evaluation steps (or several) to each iteration. With analytic derivatives, the relative time spent in an iteration step increases only as much as the derivative function increases in complexity.

In general, most of the derivatives for this formulation are independent of the specific problem being solved. In this case, since the targets always contain position constraints, not even these endpoint derivatives need to be re-derived.

In order to obtain all of these gradients linear perturbation theory must be employed. This theory posits that if the state, delineated by the vector $\vec{x}$, is perturbed along a path, $\vec{x}(t)$, at some instance $t$ along that path, this variation can be related to a corresponding variation in the initial state at the initial time (or any other arbitrary time) by use of the following equation:

$$
\delta \vec{x}(t)=\Phi\left(t, t_{0}\right) \delta \vec{x}\left(t_{0}\right)
$$

Here the matrix $\Phi\left(t, t_{0}\right)$ refers to the state transition matrix (STM), which can be obtained by integrating the state propagation matrix $F$, along with the equations of motion. The state propagation matrix (SPM) is simply the partial derivative of the time derivative of the state equation vector with respect to the state vector. 


\section{Augmented State Propagation Matrix}

In order to get the correct gradients for $\mu$, the gravitational parameter must be added to the state vector A relation between a variation in $\mu$ and the initial state of any particular segment is needed. Thus the augmented state vector becomes:

$$
\vec{x}=\left[\begin{array}{c}
\vec{r} \\
\vec{v} \\
\mu
\end{array}\right]
$$

The state propagation matrix is then:

$$
F=\frac{\partial \vec{f}}{\partial \vec{x}}=\frac{\partial \dot{\vec{x}}}{\partial \vec{x}}=\left[\begin{array}{lll}
\frac{\partial \vec{v}}{\partial \vec{r}} & \frac{\partial \vec{v}}{\partial \vec{v}} & \frac{\partial \vec{v}}{\partial \mu} \\
\frac{\partial \ddot{\vec{r}}}{\partial \vec{r}} & \frac{\partial \overrightarrow{\vec{r}}}{\partial \vec{v}} & \frac{\partial \dot{\vec{r}}}{\partial \mu} \\
\frac{\partial \dot{\mu}}{\partial \vec{r}} & \frac{\partial \dot{\mu}}{\partial \vec{v}} & \frac{\partial \dot{\mu}}{\partial \mu}
\end{array}\right]
$$

Everything in the above matrix, which depends directly on the force field propagated, has been derived before except for the last row and last column: the dependence on $\mu$. Since this algorithm is used to solve problems in both the 2-body and the CRTBP systems, the derivation for both will be given. In all derivations the time derivative rate of $\mu$ is assumed to be zero. The derivation in the 2-body frame is very simple:

$$
\begin{aligned}
& \frac{\partial \dot{\mu}}{\partial \vec{r}}=\frac{\partial \dot{\mu}}{\partial \vec{v}}=\frac{\partial \dot{\mu}}{\partial \mu}=\frac{\partial \vec{v}}{\partial \mu}=0 \\
& \frac{\partial \ddot{\vec{r}}}{\partial \mu}=-\frac{\vec{r}}{r^{3}}
\end{aligned}
$$

The augmented propagation matrix in the twobody frame is thus:

$$
F=\left[\begin{array}{ccccccc}
0 & 0 & 0 & 1 & 0 & 0 & 0 \\
0 & 0 & 0 & 0 & 1 & 0 & 0 \\
0 & 0 & 0 & 0 & 0 & 1 & 0 \\
-\frac{\mu}{r^{3}}+\frac{3 \mu x^{2}}{r^{5}} & \frac{3 \mu x y}{r^{5}} & \frac{3 \mu x z}{r^{5}} & 0 & 0 & 0 & -\frac{x}{r^{3}} \\
\frac{3 \mu y x}{r^{5}} & -\frac{\mu}{r^{3}}+\frac{3 \mu y^{2}}{r^{5}} & \frac{3 \mu y z}{r^{5}} & 0 & 0 & 0 & -\frac{y}{r^{3}} \\
\frac{3 \mu z x}{r^{5}} & \frac{3 \mu z y}{r^{5}} & -\frac{\mu}{r^{3}}+\frac{3 \mu z^{2}}{r^{5}} & 0 & 0 & 0 & -\frac{z}{r^{3}} \\
0 & 0 & 0 & 0 & 0 & 0 & 0
\end{array}\right]
$$

The same derivation in the CRTBP is a bit more complicated. The CRTBP force field equations of motion are as follows:

where:

$$
\ddot{\vec{r}}=\left[\begin{array}{c}
\ddot{x} \\
\ddot{y} \\
\ddot{z}
\end{array}\right]=\left[\begin{array}{c}
-\frac{(1-\mu)(x+\mu)}{r_{13}^{3}}-\frac{\mu(x-(1-\mu))}{r_{23}^{3}}+2 \dot{y}+x \\
-\frac{(1-\mu) y}{r_{13}^{3}}-\frac{\mu y}{r_{23}^{3}}-2 \dot{x}+y \\
-\frac{(1-\mu) z}{r_{13}^{3}}-\frac{\mu z}{r_{23}^{3}}
\end{array}\right]
$$

$$
\begin{aligned}
& r_{13}=\left[(x+\mu)^{2}+y^{2}+z^{2}\right]^{1 / 2} \\
& r_{23}=\left[(x-(1-\mu))^{2}+y^{2}+z^{2}\right]^{1 / 2}
\end{aligned}
$$

As before:

$$
\frac{\partial \dot{\mu}}{\partial \vec{r}}=\frac{\partial \dot{\mu}}{\partial \vec{v}}=\frac{\partial \dot{\mu}}{\partial \mu}=\frac{\partial \vec{v}}{\partial \mu}=0
$$

Start by finding the partials of the $\mathrm{r}$ vectors:

$$
\begin{aligned}
& \frac{\partial r_{13}}{\partial \mu}=\frac{x+\mu}{r_{13}} \\
& \frac{\partial r_{23}}{\partial \mu}=\frac{(x+(1-\mu))}{r_{23}}
\end{aligned}
$$

Then:

$$
\begin{gathered}
\frac{\partial \ddot{x}}{\partial \mu}=(x+2 \mu-1)\left[\frac{1}{r_{13}^{3}}-\frac{1}{r_{23}^{3}}\right]-\frac{3(\mu-1)(x+\mu)^{2}}{r_{13}^{5}} \\
+\frac{3 \mu(x+(1-\mu))^{2}}{r_{23}^{5}} \\
\frac{\partial \ddot{y}}{\partial \mu}=y\left[\frac{1}{r_{13}^{3}}-\frac{1}{r_{23}^{3}}\right]+\frac{3 y(\mu-1)(x+\mu)}{r_{13}^{5}} \\
+\frac{3 \mu y(x+(1-\mu))}{r_{23}^{5}} \\
\frac{\partial \ddot{z}}{\partial \mu}=z\left[\frac{1}{r_{13}^{3}}-\frac{1}{r_{23}^{3}}\right]+\frac{3 z(\mu-1)(x+\mu)}{r_{13}^{5}} \\
+\frac{3 \mu z(x+(1-\mu))}{r_{23}^{5}}
\end{gathered}
$$


The entire propagation matrix for the CRTBP is thus:

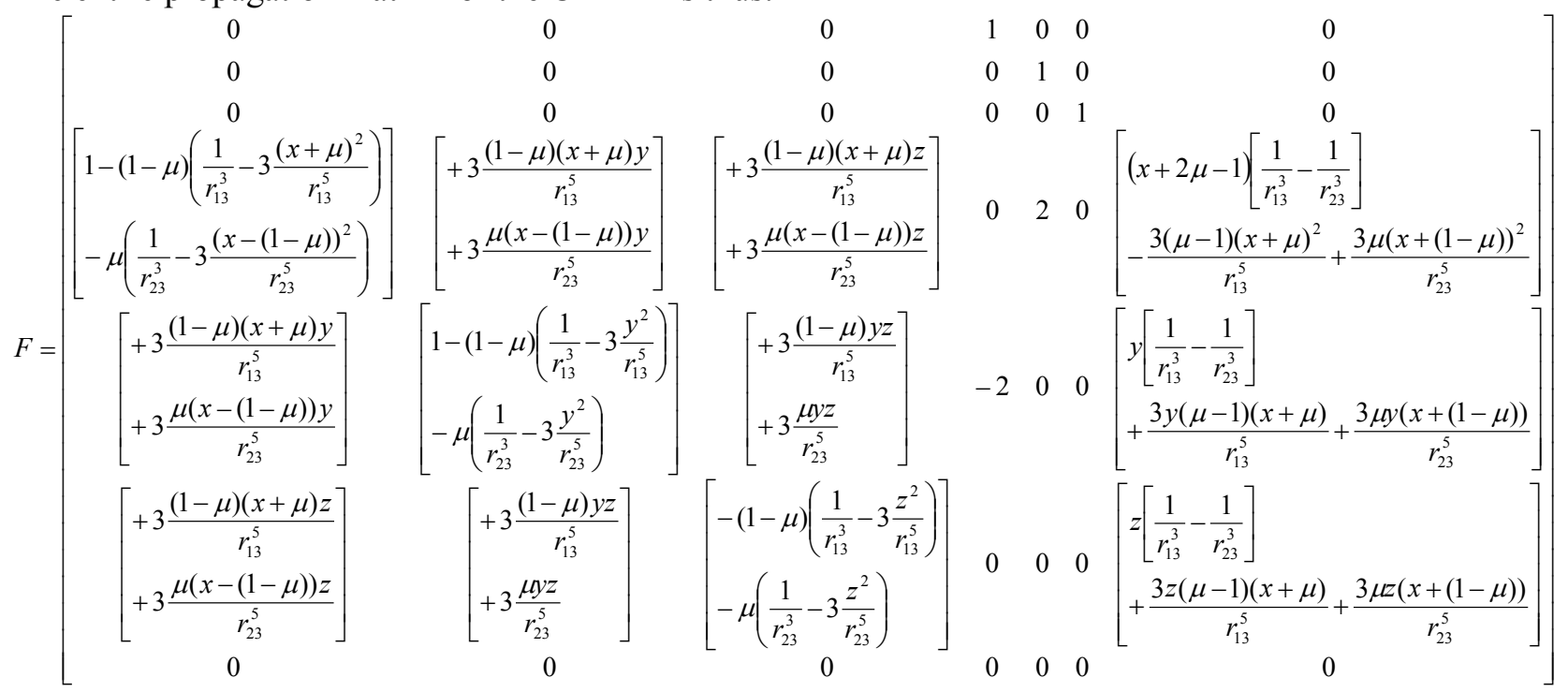

\section{Objective and Constraint Derivatives}

The usefulness of the state transition matrix found by propagating the SPM will be readily apparent after objective and constraint equations are differentiated with respect to the parameter vector.

In this derivation the same partial derivative is repeated multiple times, so only the original partial will be shown here and assumed trivial to repeat. Start with $c_{i l}$ (the first constraint ensures continuity, so the indices $i$ and $i-1$ will be used for the constraints and state vectors respectively):

$\frac{\partial c_{i 1}}{\partial x_{(i-1) 0}}=\frac{\partial\left(x_{i 0}-x_{(i-1) f}\right)}{\partial x_{(i-1) 0}}=-\frac{\partial x_{(i-1) f}}{\partial x_{(i-1) 0}}=-\Phi_{11}\left(t_{(i-1) f}, t_{(i-1) 0}\right)$

The validity of using the state transition matrix goes back to linear perturbation theory. This can be applied in every instance that a partial of a state vector of a segment at a final time is needed with respect to an initial time. The next challenging gradient is the partial derivative with respect to the initial time:

$$
\frac{\partial c_{i 1}}{\partial t_{(i-1) 0}}=\frac{\partial\left(x_{i 0}-x_{(i-1) f}\right)}{\partial t_{(i-1) 0}}=-\frac{\partial x_{(i-1) f}}{\partial t_{(i-1) 0}}
$$

The derivative at this point is not directly relatable to the STM, but the relationship between the differential and the time independent variation is known:

$$
d()=\delta()+\frac{d}{d t}() d t
$$

In terms of $x$ :

$$
d\left(x_{(i-1) f}\right)=\delta\left(x_{(i-1) f}\right)+\dot{x}_{(i-1) f} d t
$$

And can be substituted:

$$
\frac{\partial c_{i 1}}{\partial t_{(i-1) 0}}=-\frac{\partial\left(\delta\left(x_{(i-1) f}\right)+\dot{x}_{(i-1) f} d t\right)}{\partial t_{(i-1) 0}}=-\frac{\partial\left(\delta\left(x_{(i-1) f}\right)\right)}{\partial t_{(i-1) 0}}
$$

This can be simplified with the STM and another substitution of the differential (this time with $x_{(i-1))}$ ):

$$
\frac{\partial c_{i 1}}{\partial t_{(i-1) 0}}=\Phi_{11}\left(t_{(i-1) f}, t_{(i-1) 0}\right) \dot{x}_{(i-1) 0}
$$

Now evaluate with respect to the final time:

$$
\frac{\partial c_{i 1}}{\partial t_{(i-1) f}}=-v x_{(i-1) f}
$$

Compared to the previous state, the partial of this constraint to the current state $i$ is relatively trivial, it is simply the identity matrix.

The importance of augmenting the SPM is evident in the partial with respect to $\mu$ :

$\frac{\partial c_{i 1}}{\partial \mu}=\frac{\partial\left(x_{i 0}-x_{(i-1) f}\right)}{\partial \mu}=-\frac{\partial x_{(i-1) f}}{\partial \mu}=-\Phi_{17}\left(t_{(i-1) f}, t_{(i-1) 0}\right)$

The $\tau$ 's only come into play with the end point constraints and are also trivial:

$$
\frac{\partial\left(x_{i n i}-x_{10}\right)}{\partial \tau_{0}}=v x_{i n i}\left(\tau_{0}\right)
$$

And:

$$
\frac{\partial\left(x_{n f}-x_{t a r}\right)}{\partial \tau_{0}}=-v x_{t a r}\left(\tau_{f}\right)
$$


The full derivative matrix can now be created for $\mathrm{n}$ segments:

\begin{tabular}{|c|c|c|c|c|c|c|c|c|c|c|c|c|c|c|c|c|c|c|c|c|}
\hline$\vec{x}_{p}$ & $x_{10}$ & $y_{10}$ & $z_{10}$ & $v x_{10}$ & $v y_{10}$ & $v z_{10}$ & $t_{10}$ & $t_{1 f}$ & $8(n-2)$ & $x_{n 0}$ & $y_{n 0}$ & $z_{n 0}$ & $v x_{n 0}$ & $v y_{n 0}$ & $v z_{n 0}$ & $t_{n 0}$ & $t_{n f}$ & $\mu$ & $\tau_{0}$ & $\tau_{f}$ \\
\hline \multirow{18}{*}{$\frac{\partial \vec{c}}{\partial \vec{x}_{p}}=$} & $-\Phi_{11}^{1}$ & $-\Phi_{12}^{1}$ & $-\Phi_{13}^{1}$ & $-\Phi_{14}^{1}$ & $-\Phi_{15}^{1}$ & $-\Phi_{16}^{1}$ & $\Phi^{1} \dot{x}_{10}$ & $-v x_{1 f}$ & $\ldots$ & 0 & 0 & 0 & 0 & 0 & 0 & 0 & 0 & $-\Phi_{17}^{1}$ & 0 & 0 \\
\hline & $-\Phi_{21}^{1}$ & $-\Phi_{22}^{1}$ & $-\Phi_{23}^{1}$ & $-\Phi_{24}^{1}$ & $-\Phi_{25}^{1}$ & $-\Phi_{26}^{1}$ & $\Phi^{1} \dot{y}_{10}$ & $-v y_{1 f}$ & $\ldots$ & 0 & 0 & 0 & 0 & 0 & 0 & 0 & 0 & $-\Phi_{27}^{1}$ & 0 & 0 \\
\hline & $-\Phi_{31}^{1}$ & $-\Phi_{32}^{1}$ & $-\Phi_{33}^{1}$ & $-\Phi_{34}^{1}$ & $-\Phi_{35}^{1}$ & $-\Phi_{36}^{1}$ & $\Phi^{1} \dot{z}_{10}$ & $-v z_{1 f}$ & $\ldots$ & 0 & 0 & 0 & 0 & 0 & 0 & 0 & 0 & $-\Phi_{37}^{1}$ & 0 & 0 \\
\hline & $-\Phi_{41}^{1}$ & $-\Phi_{42}^{1}$ & $-\Phi_{43}^{1}$ & $-\Phi_{44}^{1}$ & $-\Phi_{45}^{1}$ & $-\Phi_{46}^{1}$ & $\Phi^{1} \dot{v} x_{10}$ & $-\dot{v} x_{1 f}$ & $\ldots$ & 0 & 0 & 0 & 0 & 0 & 0 & 0 & 0 & $-\Phi_{47}^{1}$ & 0 & 0 \\
\hline & $-\Phi_{51}^{1}$ & $-\Phi_{52}^{1}$ & $-\Phi_{53}^{1}$ & $-\Phi_{54}^{1}$ & $-\Phi_{55}^{1}$ & $-\Phi_{56}^{1}$ & $\Phi^{1} \dot{v} y_{10}$ & $-\dot{v} y_{1 f}$ & $\ldots$ & 0 & 0 & 0 & 0 & 0 & 0 & 0 & 0 & $-\Phi_{57}^{1}$ & 0 & 0 \\
\hline & $-\Phi_{61}^{1}$ & $-\Phi_{62}^{1}$ & $-\Phi_{63}^{1}$ & $-\Phi_{64}^{1}$ & $-\Phi_{65}^{1}$ & $-\Phi_{66}^{1}$ & $\Phi^{1} \dot{v} z_{10}$ & $-\dot{v} z_{1 f}$ & $\ldots$ & 0 & 0 & 0 & 0 & 0 & 0 & 0 & 0 & $-\Phi_{67}^{1}$ & 0 & 0 \\
\hline & 0 & 0 & 0 & 0 & 0 & 0 & 0 & -1 & $\ldots$ & 0 & 0 & 0 & 0 & 0 & 0 & 0 & 0 & 0 & 0 & 0 \\
\hline & 0 & 0 & 0 & 0 & 0 & 0 & -1 & 1 & $\ldots$ & 0 & 0 & 0 & 0 & 0 & 0 & 0 & 0 & 0 & 0 & 0 \\
\hline & $\vdots$ & $\vdots$ & $\vdots$ & $\vdots$ & $\vdots$ & $\vdots$ & $\vdots$ & $\vdots$ & $\ddots$ & $\vdots$ & $\vdots$ & $\vdots$ & $\vdots$ & $\vdots$ & $\vdots$ & $\vdots$ & $\vdots$ & $\vdots$ & $\vdots$ & $\vdots$ \\
\hline & 0 & 0 & 0 & 0 & 0 & 0 & 0 & 0 & $\ldots$ & 0 & 0 & 0 & 0 & 0 & 0 & -1 & 1 & 0 & 0 & 0 \\
\hline & 0 & 0 & 0 & 0 & 0 & 0 & 0 & 0 & $\ldots$ & 0 & 0 & 0 & 0 & 0 & 0 & 0 & 0 & -1 & 0 & 0 \\
\hline & -1 & 0 & 0 & 0 & 0 & 0 & 0 & 0 & $\ldots$ & 0 & 0 & 0 & 0 & 0 & 0 & 0 & 0 & 0 & $v x_{i n \tau_{0}}$ & 0 \\
\hline & 0 & -1 & 0 & 0 & 0 & 0 & 0 & 0 & $\ldots$ & 0 & 0 & 0 & 0 & 0 & 0 & 0 & 0 & 0 & $v y_{i n i \tau_{0}}$ & 0 \\
\hline & 0 & 0 & -1 & 0 & 0 & 0 & 0 & 0 & $\ldots$ & 0 & 0 & 0 & 0 & 0 & 0 & 0 & 0 & 0 & $v z_{i n i i_{0}}$ & 0 \\
\hline & 0 & 0 & 0 & 0 & 0 & 0 & 0 & 0 & $\ldots$ & $\Phi_{11}^{n}$ & $\Phi_{12}^{n}$ & $\Phi_{13}^{n}$ & $\Phi_{14}^{n}$ & $\Phi_{15}^{n}$ & $\Phi_{16}^{n}$ & $-\Phi^{n} \dot{x}_{n 0}$ & $v x_{n f}$ & $\Phi_{17}^{n}-\Phi_{17}^{\tau_{f}}$ & 0 & $-v x_{\text {tar } \tau}$ \\
\hline & 0 & 0 & 0 & 0 & 0 & 0 & 0 & 0 & $\ldots$ & $\Phi_{21}^{n}$ & $\Phi_{22}^{n}$ & $\Phi_{23}^{n}$ & $\Phi_{24}^{n}$ & $\Phi_{25}^{n}$ & $\Phi_{26}^{n}$ & $-\Phi^{n} \dot{y}_{n 0}$ & $v y_{n f}$ & $\Phi_{27}^{n}-\Phi_{27}^{\tau_{f}}$ & 0 & $-v y_{\text {tar } \tau}$ \\
\hline & 0 & 0 & 0 & 0 & 0 & 0 & 0 & 0 & $\ldots$ & $\Phi_{31}^{n}$ & $\Phi_{32}^{n}$ & $\Phi_{33}^{n}$ & $\Phi_{34}^{n}$ & $\Phi_{35}^{n}$ & $\Phi_{36}^{n}$ & $-\Phi^{n} \dot{z}_{n 0}$ & $v z_{n f}$ & $\Phi_{37}^{n}-\Phi_{37}^{\tau_{f}}$ & 0 & $-v z_{\text {tart }}$ \\
\hline & 0 & 0 & 0 & 0 & 0 & 0 & 1 & 0 & $\ldots$ & 0 & 0 & 0 & 0 & 0 & 0 & 0 & 0 & 0 & 0 & 0 \\
\hline
\end{tabular}

Notes : $\Phi^{1}=\Phi\left(t_{1 f}, t_{10}\right) \quad \Phi^{n}=\Phi\left(t_{n f}, t_{n 0}\right) \quad \Phi^{\tau_{f}}=\Phi\left(\tau_{f}, 0\right)$

The potential for mistakes is obvious, so all of these gradients were verified by numerical approximations to within a desired tolerance.

The objective gradients can be obtained in a similar manner. Recall that:

$$
\begin{gathered}
J=\left[\begin{array}{c}
\left(v x_{i n i}-v x_{10}\right)^{2}+\left(v y_{i n i}-v y_{10}\right)^{2} \\
+\left(v z_{i n i}-v z_{10}\right)^{2}
\end{array}\right]^{1 / 2}+ \\
{\left[\begin{array}{c}
\left(v x_{n f}-v x_{t a r}\right)^{2}+\left(v y_{n f}-v y_{t a r}\right)^{2} \\
+\left(v z_{n f}-v z_{t a r}\right)^{2}
\end{array}\right]}
\end{gathered}
$$

Then the objective derivatives are:

$$
\vec{x}_{p}=\left[\begin{array}{c}
x_{10} \\
y_{10} \\
z_{10} \\
v x_{10} \\
v y_{10} \\
v z_{10} \\
t_{10} \\
t_{1 f} \\
\vdots
\end{array}\right] \frac{d J}{d \vec{x}_{p}}=\left[\begin{array}{c}
0 \\
0 \\
0 \\
\left(v x_{10}-v x_{i n i}\right) / \Delta v_{0} \\
\left(v y_{10}-v y_{i n i}\right) / \Delta v_{0} \\
\left(v z_{10}-v z_{i n i}\right) / \Delta v_{0} \\
0 \\
0 \\
\vdots
\end{array}\right]
$$

Objective derivatives (cont):

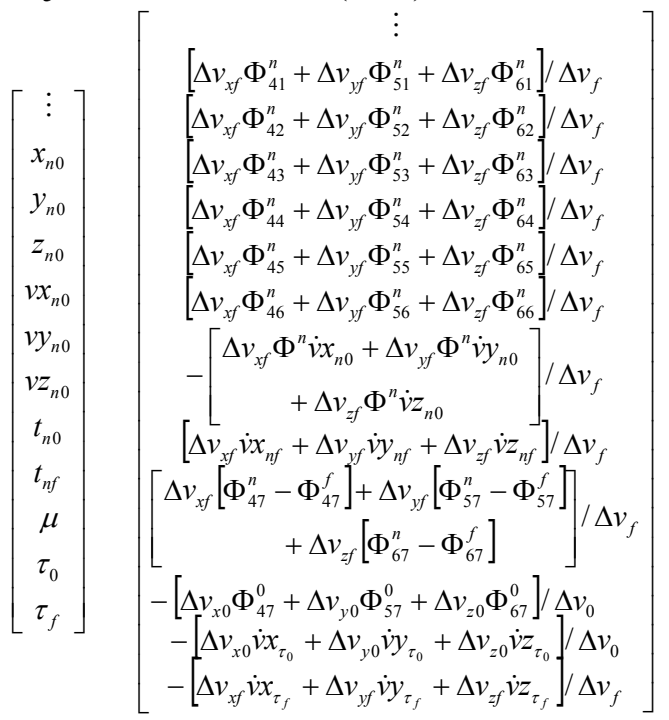

\section{Primer Vector Theory}

The optimal control theory that is used to develop primer vector theory has been presented many times, so only a summary of the aspects used in this optimization scheme are given here.

The theory that applies to this particular case is restricted to an objective function that sums impulsive velocity increments, in this case, at the endpoints. It has been shown, in both the two-body system and the CRTBP system that the primer vector and primer vector rate adhere to the same 
equations as the state variations. For the two-body system this means that:

$$
\left[\begin{array}{c}
\dot{\vec{p}} \\
\ddot{\vec{p}}
\end{array}\right]=\left[\begin{array}{cc}
0 & I \\
G & 0
\end{array}\right]\left[\begin{array}{c}
\vec{p} \\
\dot{\vec{p}}
\end{array}\right] \quad \text { or } \quad \ddot{\vec{p}}=G \vec{p}
$$

Likewise for the CRTBP:

$$
\left[\begin{array}{c}
\dot{\vec{p}} \\
\ddot{\vec{p}}
\end{array}\right]=\left[\begin{array}{cc}
0 & I \\
G_{c} & H_{c}
\end{array}\right]\left[\begin{array}{c}
\vec{p} \\
\dot{\vec{p}}
\end{array}\right]
$$

Where $\mathrm{G}_{\mathrm{c}}$ is not the same matrix as $\mathrm{G}$.

These equations make it possible to solve for the primer vector and the primer vector rate in terms of a given state and the STM that is used to transfer it:

$$
\left[\begin{array}{c}
\vec{p}(t) \\
\dot{\vec{p}}(t)
\end{array}\right]=\left[\begin{array}{cc}
\Phi_{11}\left(t, t_{0}\right) & \Phi_{12}\left(t, t_{0}\right) \\
\Phi_{21}\left(t, t_{0}\right) & \Phi_{22}\left(t, t_{0}\right)
\end{array}\right]\left[\begin{array}{c}
\vec{p}\left(t_{0}\right) \\
\dot{\vec{p}}\left(t_{0}\right)
\end{array}\right]
$$

This equation is useful if relationships for at least two out of the four quantities in the above equation are available. Fortunately, the primer vector is relatable to known values of the state by Lawden's necessary conditions for an optimal trajectory:

$$
\vec{p}\left(t_{0}\right)=\frac{\Delta \vec{v}_{0}}{\Delta v_{0}} \quad \vec{p}\left(t_{f}\right)=\frac{\Delta \vec{v}_{f}}{\Delta v_{f}}
$$

With these quantities known, the above equation is now a two point boundary value problem, which can be solved assuming the inverse of the submatrix in the upper right of the STM exists:

$$
\begin{aligned}
& \dot{\vec{p}}_{0}=\Phi_{12}\left(t_{0}, t_{f}\right)^{-1}\left[\vec{p}_{f}-\Phi_{11}\left(t_{0}, t_{f}\right) \vec{p}_{0}\right] \\
& \dot{\vec{p}}_{f}=\Phi_{21}\left(t_{0}, t_{f}\right) \vec{p}_{0}-\Phi_{22}\left(t_{0}, t_{f}\right) \dot{\vec{p}}_{0}
\end{aligned}
$$

With these quantities and the STM, the primer vector at any time $t$ can be solved for.

Other important quantities include the Hamiltonian. While in general the expressions are different for the two-body system and the CRTBP system, they can be simplified to to the same expression, given below:

$$
H=-\dot{\vec{p}} \vec{v}+\vec{p} \vec{g}
$$

On an optimal arc this should be constant 0 .

\section{KNITRO: NLP Code Usage}

All the solutions shown in this paper are found using the KNITRO nonlinear programming (NLP) algorithm. KNITRO, in its current form, is a package of three separate but not entirely distinct algorithms. They are, as named by KNITRO, an interior point conjugate gradient (CG) algorithm, an interior direct algorithm, and an SLP-EQP active set algorithm.

Of these three, the interior direct method was the most successful and efficient in solving these problems. In fact, a rigorous study comparing KNITRO with another optimizer, SNOPT, resulted in an almost exclusive use of this algorithm. To explain the differences in general terms, the interior direct method is more or less an expansion of the interior $C G$ that falls back into $C G$ iterations as necessary. And essentially, the interior point CG algorithm "is a barrier method in which the subproblems are solved approximately by an SQP iteration with trust regions" (Byrd and Hribar 878).

The main disadvantage of interior $C G$ that motivates the design of the interior direct method is the use of a conjugate gradient iteration in the calculation of the tangential step. Basically, this iteration can be very expensive if the Hessian is illconditioned, in which case a direct linear algebra technique is better. Interior direct tackles this problem by introducing a line search method in place of this CG step. However, line searches are sometimes defective due to a non-convex quadratic model and rank deficiency of the Hessian. As a result, the interior direct method simply switches from a line search approach to the interior $\mathrm{CG}$ approach above as needed. This switch is advantageous because $\mathrm{CG}$ is uniquely equipped to deal with rank deficiencies due to its use of trust regions.

\section{Solution Cases}

The various solutions shown here will be presented progressively as the complexity in the type of problem being solved is increased.

\section{2-Body Orbit Transfer}

In order to verify the algorithm the first type of problem solved was a familiar orbit transfer in the two body frame. The following table shows the values for the two orbits involved in the transfer:

Table 1: Two body Orbit Data

\begin{tabular}{cccccc}
\hline Orbit & $\mathrm{a}$ & $\mathrm{e}$ & $\mathrm{i}$ & $\Omega$ & $\omega$ \\
\hline Initial & 20000 & .2 & $0^{\circ}$ & $0^{\circ}$ & $45^{\circ}$ \\
Final & 40000 & .2 & $45^{\circ}$ & 0 & $0^{\circ}$ \\
\hline
\end{tabular}


Here is the resulting solution:

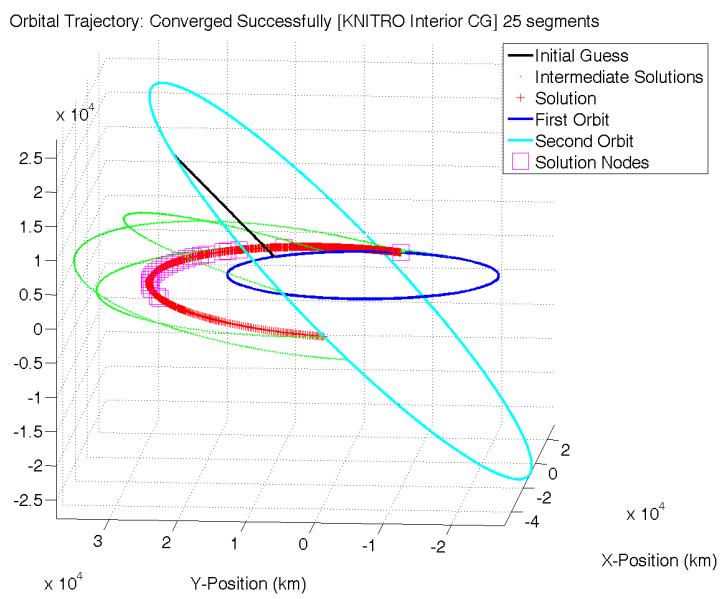

Figure 1: Two-body system orbit transfer solution

The solution above was completed with 25 segments. The initial guess is shown to be the black straight line between the dark blue and light blue orbits and the intermediate solutions that led to the final solution are shown in green. The final solution is red with each of the 25 individual segment nodes initial points indicated by squares. Here is a table for the final solution values:

Table 2: Two body Orbit Transfer Results

\begin{tabular}{cccc}
\hline Parameter & Symbol & Value & Units \\
\hline Departure & $v_{0}$ & -67.065 & degrees \\
Arrival & $v_{\mathrm{f}}$ & 181.782 & degrees \\
Flight Time & TOF & 29017.99 & seconds \\
Initial Incr. & $\Delta v_{0}$ & .7828 & $\mathrm{~km} / \mathrm{s}$ \\
Final Incr. & $\Delta v_{f}$ & 1.744 & $\mathrm{~km} / \mathrm{s}$ \\
Total Incr. & $\Delta v_{\text {total }}$ & 2.527 & $\mathrm{~km} / \mathrm{s}$ \\
\hline
\end{tabular}

The solution is locally optimal, as verified by Lawden's necessary conditions.

There are a number of things to note about this solution. First, the solution was obtained with the slightly modified approach using true anomalies instead of $\tau$ 's. Second, while 25 segments are used and successfully converge, it does not appear that 25 segments are the ideal number of segments to solve this problem. Fewer segments, if the initial conditions are tailored correctly is always more efficient. Third, while extensive knowledge of the problem does not seem to be necessary, some thought must go into the initial guess. In this case, the initial point on orbit 1 , the final point on orbit 2 , and the transfer time are the independent variables that are used to create the straight line trajectory above. Choosing different points on the orbit seems to affect what local solution is converged to and how quickly this convergence happens, while changing the transfer time can even prevent convergence. Fourth, analytic gradients decrease computation time in this problem by more than $75 \%$ but the same number of major iterations are required. Finally, the optimizer spent most of its time optimizing after quickly finding a feasible solution. In this case only 5 out of the 129 function evaluations were spent using a gravitational parameter other than the target value.

\section{CRTBP Moon Orbit to DRO Transfer}

Two impulse transfer trajectories are much more interesting in the circular restricted two body problem. The methodology described in this paper is general to any 3-body system but in this instance the Earth-Moon system was chosen, partially because of the focus of the current Constellation project on returning to the moon.

Table 3: CRTBP Moon Orbit and DRO Parameters

\begin{tabular}{ccccc}
\hline Orbit & Type & $\mathrm{x}_{0}, \mathrm{~km}$ & $\dot{y}_{0}, \mathrm{~m} / \mathrm{s}$ & $\mathrm{T}_{\mathrm{p}}$, days \\
\hline Initial & MO & 20000 & -495 & 2.9375 \\
Final & DRO & 100000 & 633.62 & 9.412 \\
\hline
\end{tabular}

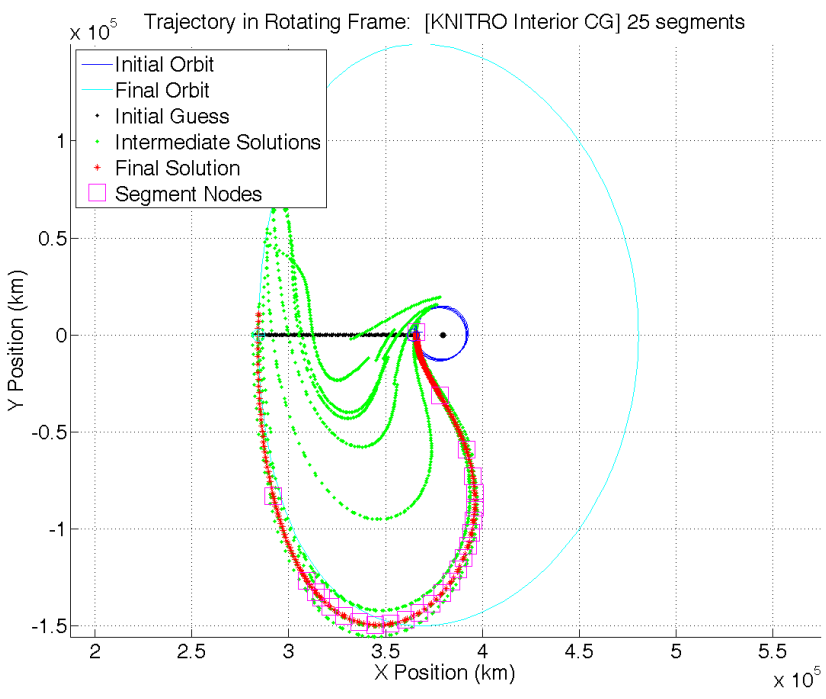

Figure 2: CRTBP DRO Transfer Solution

The initial guess for the transfer is the series of solid black line segments in the above plot. Each segment is identical to its predecessor equidistant from the selected point on the initial orbit to the point on the final orbit. While the CRTBP is a much more complicated field in which to find impulsive trajectories, this solution was found relatively easily. The primer vector history shows that this an optimal two impulse transfer: 


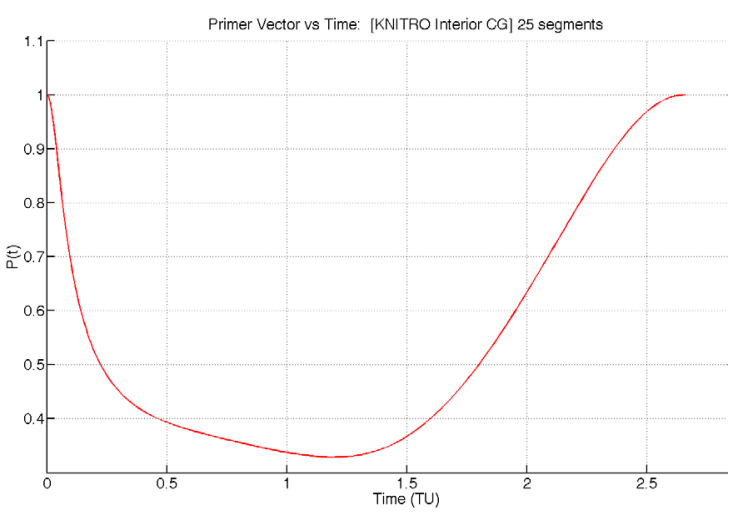

Figure 3: Primer Vector for DRO Solution

It may not be apparent in the above plot the time rate of change of the primer vector is 0 at both the initial and final time.

Table 4: CRTBP DRO Transfer Results

\begin{tabular}{cccc}
\hline Parameter & Symbol & Value & Units \\
\hline Departure & $\tau_{0}$ & 7.02 & days \\
Arrival & $\tau_{\mathrm{f}}$ & 18.82 & days \\
Flight Time & $\mathrm{TOF}$ & 11.56 & days \\
Initial Incr. & $\Delta v_{0}$ & 273.26 & $\mathrm{~m} / \mathrm{s}$ \\
Final Incr. & $\Delta v_{f}$ & 65.45 & $\mathrm{~m} / \mathrm{s}$ \\
Total Incr. & $\Delta v_{\text {total }}$ & 338.71 & $\mathrm{~m} / \mathrm{s}$ \\
\hline
\end{tabular}

Again, like in the two-body orbit transfer, fewer segments can be used to find a convergence quicker, but comparing the work it takes to acquire these solutions reveals some drawbacks to using fewer segments. In the CRTBP system a zero gravitational parameter is no longer a force-free system. Thus each segment will not end up at the starting point of the next segment; in figure 2 , each of the black segments along the solid line diverges noticeably from a straight line. The drawback is directly related to this: basically anytime an individual segment diverges significantly enough from the straight line by the dynamics the individual segments create wildly confusing guesses. By adding segments, the initial guess on transfer time can be worse, thus adding robustness. It is important to note that analytical gradients are a significant aid to reducing the solution time.

\section{CRTBP Earth Orbit to Lyapunov Orbit}

Transfers to libration point orbits such as Lyapunov and Halo orbits from initial Earth orbits can be difficult to design. Often optimal paths to these orbits are found by analyzing the unstable manifolds associated with them. These manifolds delineate paths that diverge quickly from the periodic orbits; if a spacecraft can hop on one in the vicinity of an Earth orbit the impulsive maneuver at the end of the trajectory is practically zero. The trick is finding a path that passes close to an initial parking orbit that requires a small impulse.

If this algorithm is adept at picking up these manifold arcs and can reliably find transfers to these orbits without utilizing the dynamics associated with the manifold, it bodes well for the future development of a highly robust algorithm. If anything it could be useful for discovering new novel transfers. It appears that the algorithm may be sensitive enough, as the below converged transfer to a large Lyapunov orbit around $\mathrm{L}_{1}$ demonstrates. 


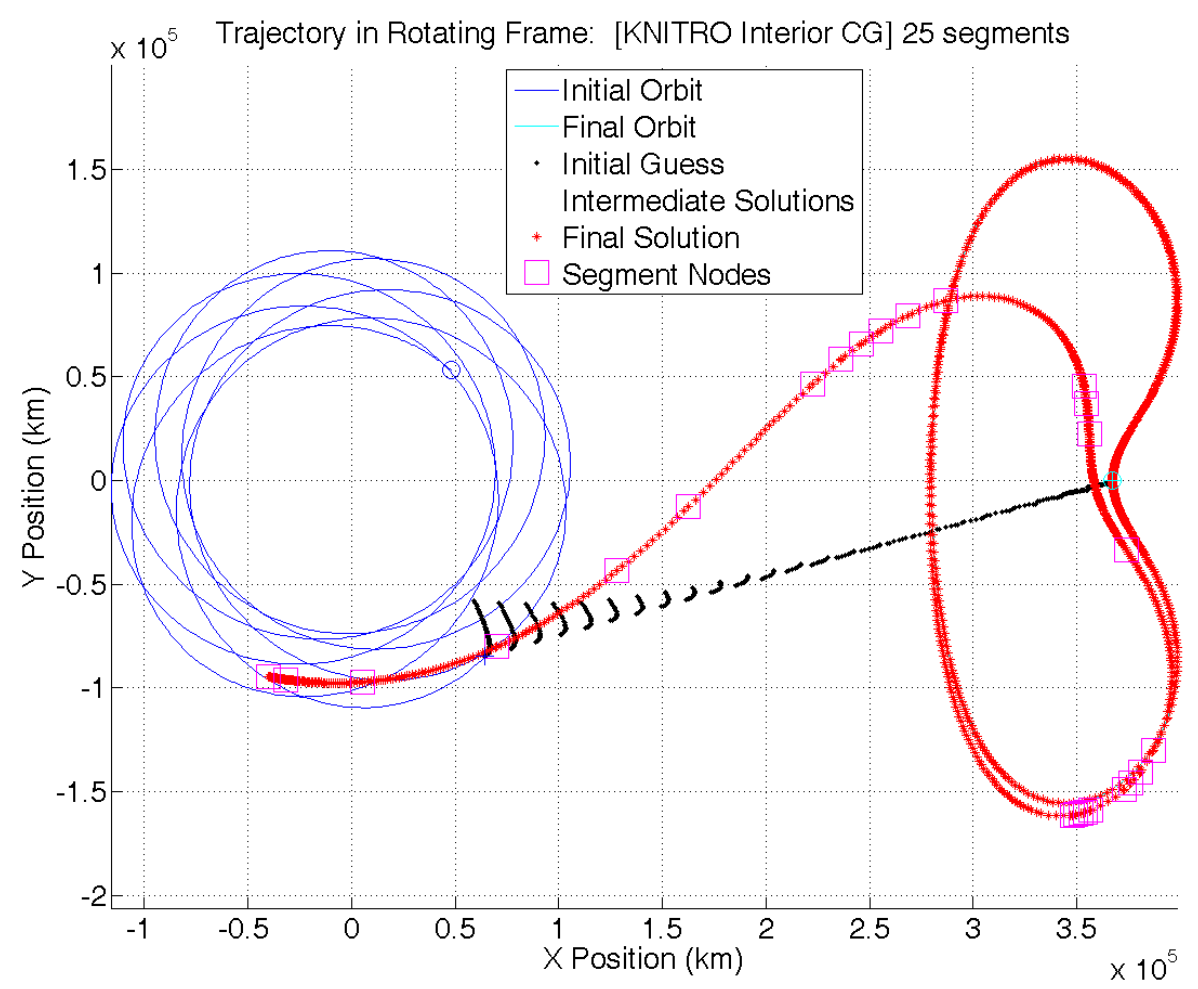

Figure 4: Optimal Transfer to Lyapunov Orbit

Table 5: Earth and Lyapunov Orbit Parameters

\begin{tabular}{cccc}
\hline Orbit & Type & $\mathrm{x}_{0}, \mathrm{~km}$ & $\dot{y}_{0}, \mathrm{~km} / \mathrm{s}$ \\
\hline Initial & EO & 75000 & 2.305 \\
Final & Lyapunov & 280000 & 0.5902 \\
\hline
\end{tabular}

There are a number of qualities to note about this trajectory. First it appears to be a local minimum, as the primer vector plot helps confirm:

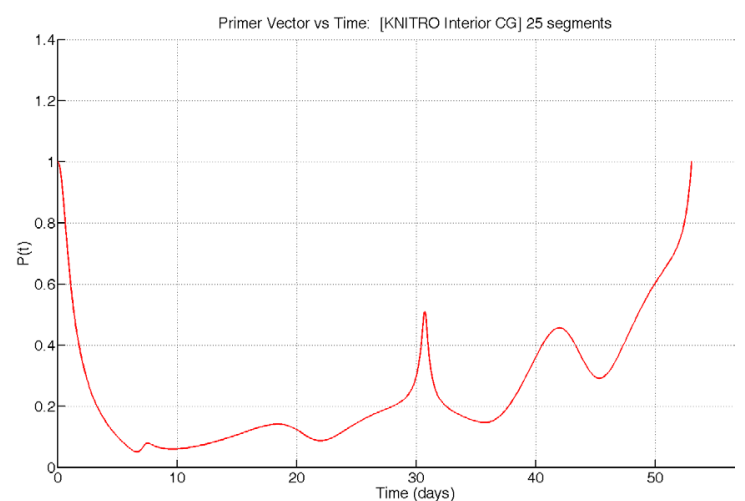

Figure 5: Primer Vector for Lyapunov Transfer

Although it is difficult to tell in figure 5, the rate is zero at the initial point. However, this is not true at the final point. It is speculated that this is due to the extremely small magnitude of the final maneuver.

Table 6: CRTBP Lyapunov Transfer Results

Parameter Symbol Value Units

\begin{tabular}{cccc} 
Departure & $\tau_{0}$ & 24.752 & days \\
Arrival & $\tau_{\mathrm{f}}$ & 22.996 & days \\
Flight Time & $\mathrm{TOF}$ & 53.062 & days \\
Initial Incr. & $\Delta v_{0}$ & 571.65 & $\mathrm{~m} / \mathrm{s}$ \\
Final Incr. & $\Delta v_{f}$ & 0.0045 & $\mathrm{~m} / \mathrm{s}$ \\
Total Incr. & $\Delta v_{\text {total }}$ & 571.65 & $\mathrm{~m} / \mathrm{s}$ \\
\hline
\end{tabular}

The initial guess was once again very important in finding a solution. The impact of the dynamics is much more obvious in the initial guess shown in figure 4. Especially near the Earth, the segments bend towards the Earth. Basically in order to get a solution the segments need to be long enough with a low enough initial velocity but not too long less they try to wrap around the primary. If this happens with the initial guess the segments split in a myriad of directions and only reconnect after many random passes around the system which is not likely to result in an optimal trajectory.

An arbitrary set of orbits will not necessarily have a single pass optimal trajectory such as this. The two dimensional nature of this orbit transfer may also be a simpler trajectory to find.

Perhaps the most interesting part about finding this solution is the need for a large number of segments. Even if the simple rules that have been established for having a good initial guess are followed as discussed in this paper, this trajectory was never found with less than 25 segments. Up until this point, fewer segments was always more 
advantageous, or seemingly so since run times could always be decreased. But it seems that having lots of segments not only makes the search more exploratory it may also keep the search well behaved. The evidence for this trend lies in the intermediate trajectories found from iteration to iteration. For fewer segments the intermediate trajectories can have very wild trajectory guesses. With 25 segments, the intermediate guesses usually stay disjointed longer but with a good initial guess the full trajectory rarely starts to make a huge jump between iterations to a radically different trajectory. The explanation might relate to the length of the segments. With only 1 long segment a small change in the initial state can make a large difference in the end point of that segment. But for each of several segments a small change has a much smaller effect on the entire trajectory. Up to this point the drawbacks for using many segments, mainly the increased run time, has outweighed the potential benefits But with analytical derivatives and a powerful NLP code, some of these drawbacks may be reduced significantly enough to make it work. This optimal trajectory is certainly a large step in that direction.

Even with this solution, however, any transfer in the CRTBP frame appears to have less room for variation in the initial guess. This challenges the notion that it is truly robust with so much of the success of solving a problem depending on the character of the initial guess.

\section{CRTBP Earth Orbit to Halo Orbit}

While there seems to be a lot of success in finding transfers in the CRTBP frame to 2-d Lyapunov orbits, Halo orbits appear to present more trouble for the algorithm. Again, some of the transfers atempted may not have a good single pass transfer if the manifold does not pass closely to the orbit being transferred from.

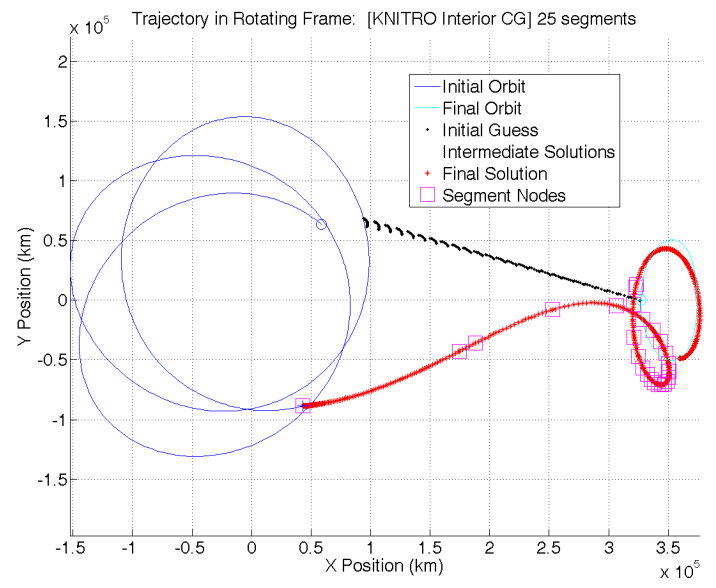

Figure 6: Transfer to Halo Orbit, Top View

However, while they may not be completely optimal, transfers to halo orbits that have very low final orbit transfer velocities can be found.

Table 7: Earth Orbit and Halo Orbit Parameters

\begin{tabular}{ccccc}
\hline Orbit & Type & $\mathrm{x}_{0}, \mathrm{~km}$ & $\mathrm{z}_{0}, \mathrm{~km}$ & $\dot{y}_{0}, \mathrm{~km} / \mathrm{s}$ \\
\hline Initial & EO & 90000 & 0 & 2.305 \\
Final & Halo & 374200 & -27910 & -.5344 \\
\hline
\end{tabular}

Trajectory in Rotating Frame: [KNITRO Interior CG] 25 segments

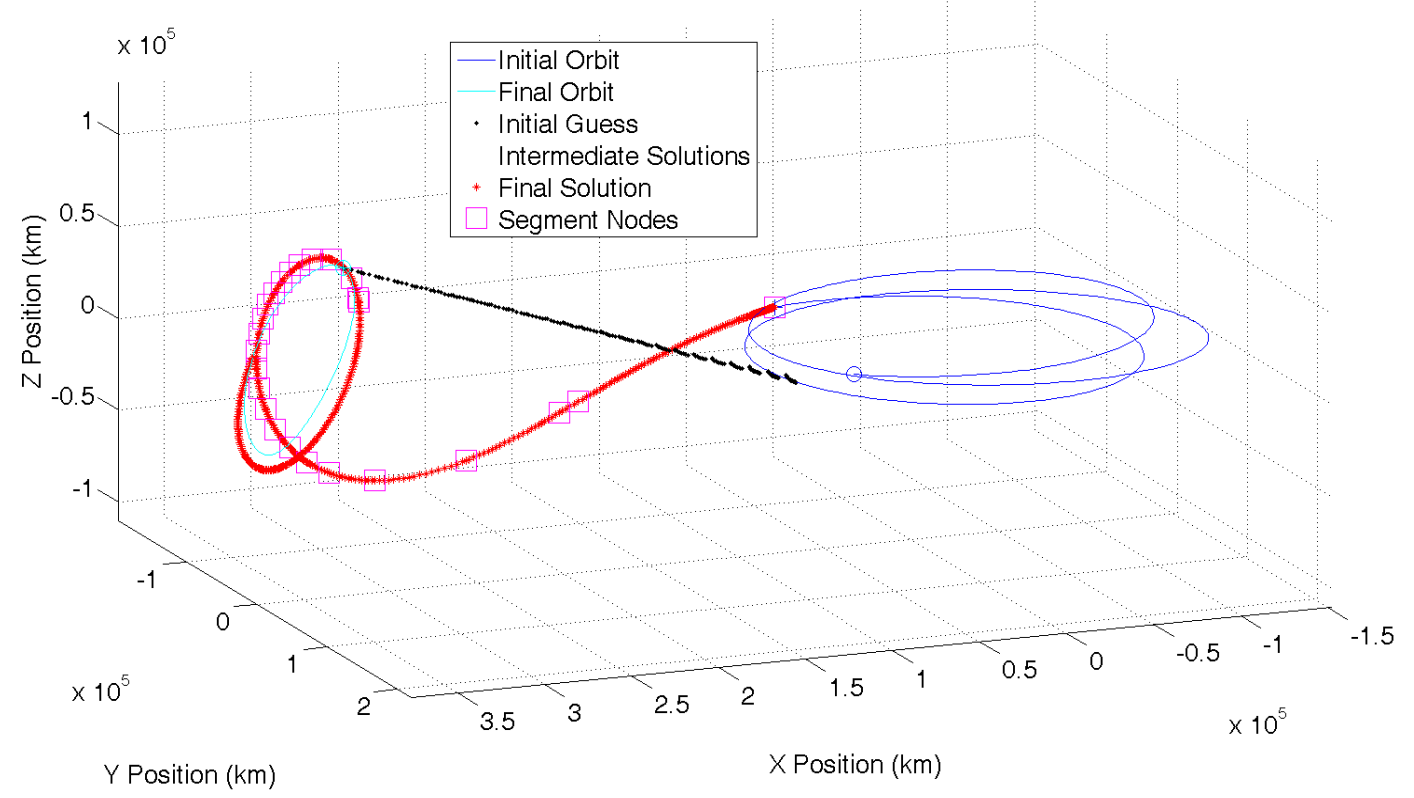

Figure 7: Transfer to Halo Orbit, 3D View 
Table 8: Earth to Halo Orbit Transfer Results

\begin{tabular}{cccc}
\hline Parameter & Symbol & Value & Units \\
\hline Departure & $\tau_{0}$ & 10.856 & days \\
Arrival & $\tau_{\mathrm{f}}$ & 10.994 & days \\
Flight Time & TOF & 19.711 & days \\
Initial Incr. & $\Delta v_{0}$ & 562.09 & $\mathrm{~m} / \mathrm{s}$ \\
Final Incr. & $\Delta v_{f}$ & 86.93 & $\mathrm{~m} / \mathrm{s}$ \\
Total Incr. & $\Delta v_{\text {total }}$ & 649.02 & $\mathrm{~m} / \mathrm{s}$ \\
\hline
\end{tabular}

The primer vector plot reveals a lot about the optimality of the solution:

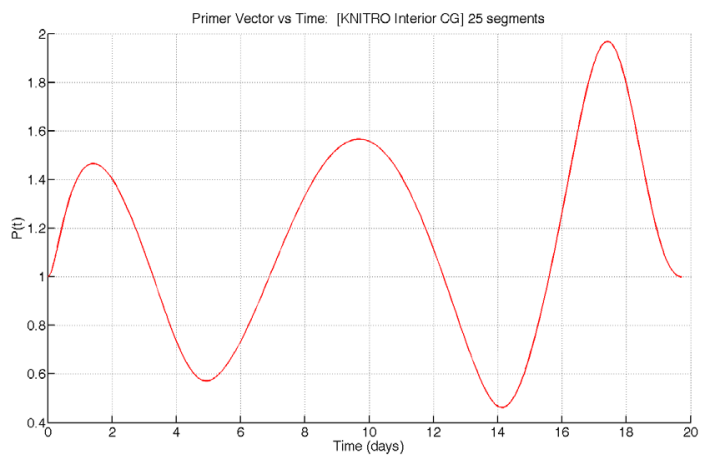

Figure 8: Primer Vector for Transfer to Halo

Clearly the primer magnitude exceeds 1 and does so at three locations. This may be an optimal two-impulse solution that simply needs intermediate impulses. This presents an intriguing modification to the algorithm. If may be possible to modify the segment constraints so that the velocity increment between segments is simply added to the objective function rather than constrained to be continuous. While the analytic derivatives would need to be rederived this would add capability to the algorithm to find multiple impulse solutions.

\section{CRTBP Halo Orbit to Halo Orbit}

Using primer vector theory to find orbit transfers is definitely not new. In fact, many of the concepts applied in this paper were used to find optimal trajectories in the CRTBP system in the available literature.

In one, transfers between Halo orbits were found. These relatively simple CRTBP transfers were likewise modeled here to help verify implementation of the theory. Without a detailed account of the trajectories being analyzed it is hard to directly reproduce the results but it appears at least the character of the solution is similar:

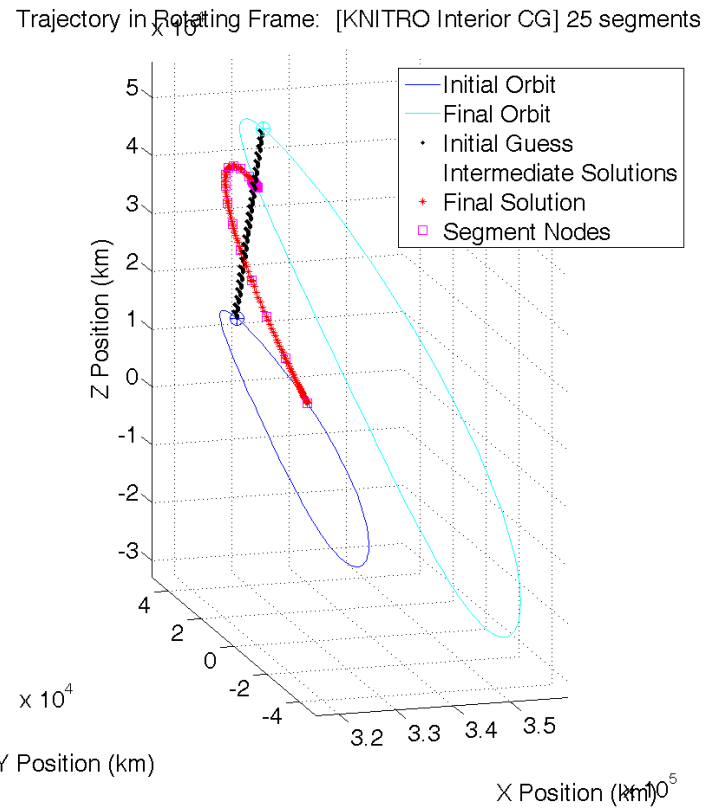

Figure 9: Halo to Halo Transfer

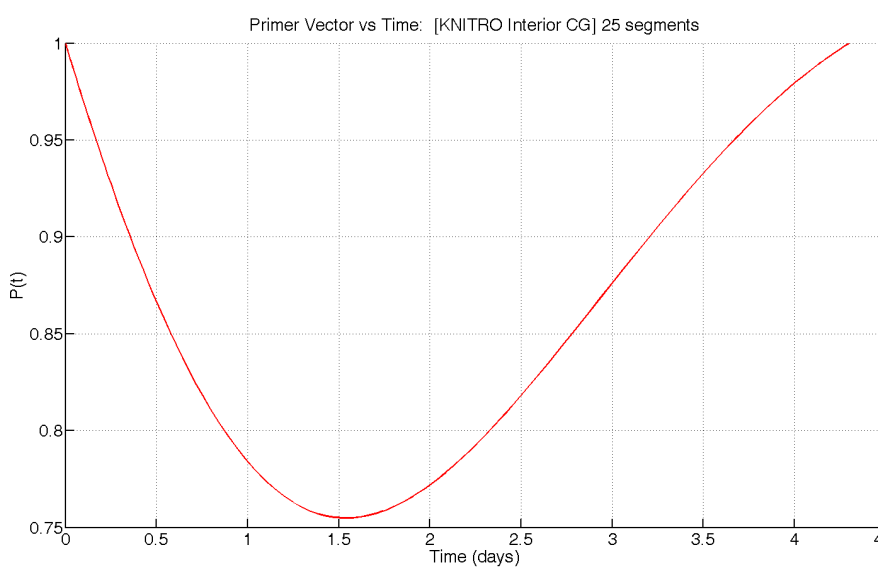

Figure 10: Primer Vector for Halo to Halo Transfer

The primer vector plot suggests that the initial and final times may need to shift earlier and later respectively as the primer rate does not go to zero at the end points. The fact that it converged may mean that the orbits are too sensitive. One remedy might be scaling.

\section{Conclusions}

The work presented here demonstrates that a new algorithm, a multiple direct shooting method that uses the gravitational parameter as an optimization variable, can be used to solve various orbital trajectory optimization problems. Some problems respond better than others to the algorithm. In the CRTBP system optimal transfers to periodic libration point orbits were the most surprising finds. 
Future modifications to the program may increase robustness further. This may be accomplished by including more advanced NLP codes, by adding the capability to handle intermediate impulses, or by designing an automated initial guess program. If the drawbacks can be ironed out, a robust algorithm may be on the horizon.

\section{References}

${ }^{1}$ Byrd, R., Hribar, M., Nocedal, J., "An interior point algorithm for large scale nonlinear programming," SIAM Journal on Optimization, 9(4):877-900, 1999.

${ }^{2}$ Byrd, R., Gould, M., Nocedal, J., and Waltz, R., “An algorithm for nonlinear optimization using linear programming and equality constrained subproblems." Mathematical Programming, Series B, 100(1):27-48, 2004.

${ }^{3}$ Farquhar, Robert W., "Halo-Orbit and Lunar-Swingby Mission of the 1990's," Acta Astronautica, Vol. 24, 1991, pp. 227-234.

${ }^{4}$ Gill, P., Murray, W., and Saunders, M., "SNOPT: An SQP algorithm for large-scale constrained optimization.” Technical Report 97-22, Dept. of Mathematics, University of California, San Diego, USA, 1997.

${ }^{5}$ Hiday-Johnston, L.A., and Howell, K.C., "Impulsive Time-Free Transfers Between Halo Orbits, "Celestial Mechanics and Dynamical Astronomy," Vol. 64, 1996, pp281-303.

${ }^{6}$ Howell, K.C., and Barden, B.T., "Using Stable Manifolds to Generate Transfers from Earth to Sun-Earth Libration Point Orbits," Advances in Nonlinear Astrodynamics Conference, The Geometry Center, University of Minnesota, Minneapolis, Minnesota, November 1993.

${ }^{7}$ Morales, J., Nocedal, J., Waltz, R., Liu, G., and Goux, J., "Assessing the potential of interior methods for nonlinear optimization." Technical report OTC 2001/04, Optimization Technology Center, Northwestern University, Evanston (2001)

${ }^{8}$ Noecedal, J., Waechter, A., and Waltz, R., "Adaptive Barrier Update Strategies for Nonlinear Interior Methods", Tech. Report, IBM Watson Research Center, 2005.

${ }^{9}$ Ocampo, C., and Rosborough, G., "Multiple-Spacecraft Orbit-Transfer Problem: The No-Booster Case," Journal of Guidance, Control, and Dynamics, Vol. 22, No. 5, 1999, pp650-657.

${ }^{10}$ Ocampo, C., and Rosborough, G., "Multiple-Spacecraft Orbit-Transfer Problem: Weak-Booster and Strong-Booster Cases," Journal of Guidance, Control, and Dynamics, Vol. 22, No. 6, 1999, pp897-904.

${ }^{11}$ Senent, J., Ocampo, C., and Capella, A., "Low-Thrust Variable-Specific-Impulse Transfers and Guidance to Unstable Periodic Orbits," Journal of Guidance, Control and Dynamics, Vol. 28, No. 2, 2005, pp 280-290.

${ }^{12}$ Waltz, R., Morales, J., Nocedal, J., and Orban, D., “An interior algorithm for nonlinear optimization that combines line search and trust region steps." Mathematical Programming A, 107(3):391-408, 2006. 